\title{
Skin friction and pressure: the "footprints" of turbulence
}

\author{
Thomas R. Bewley and Bartosz Protas \\ Flow Control Lab, Dept. of MAE, UC San Diego, La Jolla, CA 92093-0411, USA
}

\begin{abstract}
The problems of exact state reconstruction and approximate state estimation based on wall information in a wall-bounded incompressible unsteady flow are addressed. It is shown that, if in an arbitrarily small neighborhood of time $t$ precise measurements are made of the two components of wall skin friction and the wall pressure, all terms in the Taylor-series expansions of the unsteady flow state near the wall at time $t$ may be determined (in the linear setting, this determination may be made based on skin friction measurements alone). Combining this fact with the analyticity of solutions of the nonlinear Navier-Stokes equation and the unique continuation theorem for analytic functions, in theory complete reconstruction of a fully-developed turbulent flow in a channel at any Reynolds number at time $t$ is possible given only information about the unsteady flow available at the wall in a neighborhood of time $t$, without knowledge of the initial conditions of the flow. Thus, skin friction and pressure measurements on the wall in a neighborhood of time $t$ provide a unique "footprint" of the entire unsteady turbulent flow state; no other flow can have the same footprint. Indeed, higher-order terms are shown to uniformly improve the correlation of truncated Taylor-series expansions with the DNS of a turbulent flow near the wall. However, such series extrapolations amplify measurement noise, as they require differentiation in both space and time of the measurements, and the radius of convergence of the Taylor series expansions is less than 10 wall units. The so-called Linear Stochastic Estimation technique, in which the polynomials forming the basis of the series expansion are replaced by well-behaved functions (such as POD modes) on the entire flow domain also demonstrates very poor convergence. In light of these limitations on direct extrapolations from measurements in the practical setting, an adjoint-based algorithm is presented and numerically tested for estimating the state of an entire turbulent channel flow system based on a time history of noisy measurements at the wall. This algorithm effectively uses the unsteady nonlinear Navier-Stokes equation itself as a filter to find the flow solution that is most consistent with the available measurements.
\end{abstract}

\section{Introduction}

During the last 10 years, there has been a flurry of activity in controlling both laminar and turbulent flows in certain idealized settings. The goal of this research thrust has been twofold: to learn more about fundamental flow physics, and to begin to shed light on how to control fluid flow in practical engineering applications with model-based control strategies. For recent surveys of this active field of research, see, e.g., Gad-el-Hak (2001), Gunzberger (2002), and Bewley (2001), and the references contained therein. 
An important and largely unsolved problem in model-based feedback control of turbulence is the estimation of the unsteady flow state based on the available flow measurements when the initial conditions of the unsteady flow are unknown. From the literature survey we have performed (see the above-mentioned review articles for several examples), it appears that, to date, all efforts to control and/or estimate wall-bounded flows with information available at the wall only have used measurements of either wall skin friction or wall pressure. A few examples from groups working in related areas include Kravchenko, Choi, \& Moin (1993), Choi, Moin, \& Kim (1994), Rathnasingham \& Breuer (1997), Podvin \& Lumley (1998), Koumoutsakos (1999), Luchini, Bottaro, \& Zuccher (2001), and Yoshino, Tsuda, Suzuki, \& Kasagi (2002). Those who have explored the possible role of pressure measurements in flow estimation and control applications include Johansson, Her, \& Haritonidis (1987), Gunzburger \& Lee (1996), Hernandez, Baudet, \& Fauve (2000), Balogh, Liu, \& Krstic (2001), Gad-el-Hak (2001), Lee \& Sung (2002), and Kim, Choi, \& Sung (2002). The present note characterizes the additional opportunities that are available when measurements of both wall skin friction and wall pressure are used.

In $\S 2$, it is shown that, if precise measurements are made in a neighborhood of time $t$ of the two components of wall skin friction, $\partial u / \partial y$ and $\partial w / \partial y$, and the wall pressure, $p$, an arbitrary number of terms in the Taylor-series expansions of the turbulent flow state near the wall at time $t$ may be determined. Thus, at least in theory, with this information we can find the solution at time $t$ to an unsteady flow problem without the knowledge of the initial condition. Using a high-fidelity DNS database of an $R e_{\tau}=180$ turbulent channel flow, it is shown that the radius of convergence of these Taylor series appears to be less than 10 wall units.

The idea of extrapolating directly from instantaneous measurements to flowfield patterns has gained a certain level of popularity in the field of fluid mechanics. The approach commonly used, based on conditional averages, is referred to as Linear Stochastic Estimation (LSE), and is discussed further in Adrian (1977) and Cole, Glauser, \& Guezennec (1992). In the present investigation, changing the set of basis functions to an orthogonal, well-behaved set of basis functions on the entire flow domain (such as Fourier in $x$ and $z$ and Chebyshev or POD in $y$ ), in the spirit of the LSE approach, demonstrated even worse convergence properties than the approach based on Taylor series, as discussed briefly in $\$ 2.7$.

In practice, measurements are noisy, and thus dynamic state estimation strategies which filter the measured information using the governing equation itself (such as Riccati-based extended Kalman filters and adjoint-based methods for model predictive estimation) are much better behaved than ill-posed direct extrapolations of the flowfield from instantaneous measurements at the wall. Significantly, dynamic state estimation strategies assimilate the information contained in an available history of noisy measurements into an evolving estimate of the state without requiring differentiation of the measurements, thereby extracting the information in the history of noisy measurements which is most consistent with the governing equation itself. In $\$ 3$, a model predictive algorithm is presented and numerically tested for this state estimation problem. It is shown that the three types of available wall measurements (that is, $\partial u / \partial y, \partial w / \partial y$, and $p$ ) may be used to drive the three possible locations of forcing on the boundary in the relevant adjoint problem, and numerical simulations again indicate the utility of simultaneously using all three flow quantities available at the wall when attempting to do practical state estimation. 
Note that referring to the boundary values of $\partial u / \partial y$ and $\partial w / \partial y$ as "wall skin friction" is a bit loose, as the corresponding components of the shear-stress tensor at the wall, $\tau_{x y}=\mu(\partial u / \partial y+\partial v / \partial x)$ and $\tau_{z y}=\mu(\partial w / \partial y+\partial v / \partial z)$, both include contributions from the boundary values of $v$ on the wall and are scaled by the viscosity $\mu$. We assume the viscosity $\mu$ and the wall-normal velocity $v$ at the wall are known in this work, so $\partial u / \partial y$ and $\partial w / \partial y$ may easily be determined from measurements of $\tau_{x y}$ and $\tau_{z y}$ at the wall. The idealized problem of a continuous distribution of both actuation and sensing on the wall is not physically realizable anyway; how this configuration might be approximated in a real implementation is an application-specific issue which we will not address here. We will thus use the words "streamwise and spanwise wall skin friction distributions" to refer to the distributions of $\partial u / \partial y$ and $\partial w / \partial y$ on the wall without ambiguity.

\subsection{Governing equations}

This paper considers an incompressible unsteady flow in a channel with known Dirichlet boundary conditions on the velocity, $\left\{u_{w}, v_{w}, w_{w}\right\}$, known (and sufficiently smooth) externally-applied forcing $\left\{F_{1}, F_{2}, F_{3}\right\}$ on the interior, and known measurements of the skin-friction and pressure dis-

tributions on the walls, $\left\{\left.\frac{\partial u}{\partial y}\right|_{w},\left.p\right|_{w},\left.\frac{\partial w}{\partial y}\right|_{w}\right\}$. Initial conditions on the flow at time $t_{0}$ are unknown; we desire to exactly reconstruct (or approximately estimate) the unsteady flow state at time $t>t_{0}$ everywhere in the channel based on the wall information and the externally-applied forcing only.

Without loss of generality, $\$ 2$ analyzes the region adjacent to one of the walls, defining the $x-y-z$ coordinate system such that $y$ is the wall-normal direction, with the wall located at $y=0$. In $\S 3$, we switch to an $x_{1}-x_{2}-x_{3}$ coordinate system, and consider an entire channel-flow system in the domain $\left(0 \times L_{1}\right) \times(-1 \times 1) \times\left(0 \times L_{3}\right)$.

The Navier-Stokes equation governing the flow is given by

$$
\begin{gathered}
\frac{\partial u}{\partial t}=-\frac{\partial p}{\partial x}+v \Delta u+F_{1}-u \frac{\partial u}{\partial x}-v \frac{\partial u}{\partial y}-w \frac{\partial u}{\partial z} \\
\frac{\partial v}{\partial t}=-\frac{\partial p}{\partial y}+v \Delta v+F_{2}-u \frac{\partial v}{\partial x}-v \frac{\partial v}{\partial y}-w \frac{\partial v}{\partial z} \\
\frac{\partial w}{\partial t}=-\frac{\partial p}{\partial z}+v \Delta w+F_{3}-u \frac{\partial w}{\partial x}-v \frac{\partial w}{\partial y}-w \frac{\partial w}{\partial z} \\
0=\frac{\partial u}{\partial x}+\frac{\partial v}{\partial y}+\frac{\partial w}{\partial z}
\end{gathered}
$$

where $\Delta \triangleq \partial^{2} / \partial x^{2}+\partial^{2} / \partial y^{2}+\partial^{2} / \partial z^{2}$. The continuity equation (1.2) constrains the three velocity components $\{u, v, w\}$, which evolve according to the momentum equations (1.1a)-(1.1c), to lie in a divergence-free subspace. This constraint is applied through the influence of the pressure $p$ in the momentum equations, which acts as a Lagrange multiplier in these equations in such a way that the continuity equation is satisfied at every point in space and every instant in time. We thus see that the Navier-Stokes equation effectively admits only two degrees of freedom per spatial location. Noting this fact, it is common to represent solutions to incompressible Navier-Stokes systems with a reduced, divergence-free form, thus applying the continuity equation implicitly. 
One popular divergence-free form, convenient in terms of the imposition of Dirichlet boundary conditions on the velocity at the walls in a plane channel flow, is the $\left\{v, \omega_{y}\right\}$ form, in which the wallnormal component of velocity, $v$, and the wall-normal component of vorticity, $\omega_{y} \triangleq \partial u / \partial z-\partial w / \partial x$, are retained as the two independent degrees of freedom per spatial location. From these two fields and the appropriate boundary conditions, $u$ and $w$ may be reconstructed exactly, and $p$ may be determined up to an arbitrary constant. In the $\left\{v, \omega_{y}\right\}$ formulation, evolution equations governing $v$ and $\omega_{y}$ are found by appropriate manipulation of (1.1) and (1.2). The right-hand sides of these equations may be interpreted as functions of $v$ and $\omega_{y}$ only by substitution of the appropriate formulae for the reconstructions of $u, w$, and $p$. The fact that only two of the four variables in the set $\{u, v, w, p\}$ are independent in incompressible flows can lead to the mistaken impression that wall measurements of $\partial u / \partial y, \partial w / \partial y$, and $p$ must in some sense be redundant. Though this is in fact true in the linear case, this is not true in the nonlinear case, as shown below.

\section{Exact state reconstruction from precise wall information}

\subsection{Taylor-series approximation: the general case}

The Taylor-series expansions near the wall of the individual components of the velocity and the pressure may be written in the form

$$
\begin{aligned}
& u(x, y, z, t)=\sum_{j=0}^{\infty} a_{j} \frac{y^{j}}{j !}, \quad v(x, y, z, t)=\sum_{j=0}^{\infty} b_{j} \frac{y^{j}}{j !}, \quad w(x, y, z, t)=\sum_{j=0}^{\infty} c_{j} \frac{y^{j}}{j !}, \quad p(x, y, z, t)=\sum_{j=0}^{\infty} d_{j} \frac{y^{j}}{j !}, \\
& a_{j}(x, z, t)=\left.\frac{\partial^{j} u}{\partial y^{j}}\right|_{w}, \quad b_{j}(x, z, t)=\left.\frac{\partial^{j} v}{\partial y^{j}}\right|_{w}, \quad c_{j}(x, z, t)=\left.\frac{\partial^{j} w}{\partial y^{j}}\right|_{w}, \quad d_{j}(x, z, t)=\left.\frac{\partial^{j} p}{\partial y^{j}}\right|_{w} .
\end{aligned}
$$

Taylor-series expansions may be defined in a similar fashion for the individual components of the vorticity, with expansion coefficients $\left\{e_{j}, f_{j}, g_{j}\right\}$. We now seek to express the expansion coefficients $\left\{a_{j}, b_{j}, c_{j}, d_{j}, e_{j}, f_{j}, g_{j}\right\}$ as a function of the externally-applied forcing, $\left\{F_{1}, F_{2}, F_{3}\right\}$, and the available data on the wall, which includes the boundary conditions on the velocity $\left\{u_{w}, v_{w}, w_{w}\right\}$ and the measurements $\left\{\left.M_{1} \triangleq \frac{\partial u}{\partial y}\right|_{w},\left.M_{2} \triangleq p\right|_{w},\left.M_{3} \triangleq \frac{\partial w}{\partial y}\right|_{w}\right\}$.

We first observe that computing $\partial^{j} / \partial y^{j}$ of (1.2) results in $b_{j+1}=-\partial a_{j} / \partial x-\partial c_{j} / \partial z$; that is, higherorder expansion coefficients for $v$ may be expressed as a simple function of lower-order expansion coefficients for $u$ and $w$. We note also that the zeroth- and first-order expansion coefficients for $u$ and $w$ and the zeroth-order expansion coefficient for $v$ and $p$ are given directly by the boundary conditions and measurements. We therefore have

$$
\begin{aligned}
& a_{0}=u_{w}, \quad b_{0}=v_{w}, \quad c_{0}=w_{w} \\
& a_{1}=M_{1}, \quad b_{1}=-\partial a_{0} / \partial x-\partial c_{0} / \partial z, \quad c_{1}=M_{3}, \quad d_{0}=M_{2} \text {. }
\end{aligned}
$$

The second-order expansion coefficients for $u$ and $w$ and the first-order expansion coefficient for $p$ 
may be obtained by rearranging the momentum equations (1.1) in the following form:

$$
\begin{aligned}
\frac{\partial^{2} u}{\partial y^{2}} & =\frac{1}{v}\left[\frac{\partial u}{\partial t}+\frac{\partial p}{\partial x}-v \Delta_{s} u-F_{1}+u \frac{\partial u}{\partial x}+v \frac{\partial u}{\partial y}+w \frac{\partial u}{\partial z}\right], \\
\frac{\partial^{2} w}{\partial y^{2}} & =\frac{1}{v}\left[\frac{\partial w}{\partial t}+\frac{\partial p}{\partial z}-v \Delta_{s} w-F_{3}+u \frac{\partial w}{\partial x}+v \frac{\partial w}{\partial y}+w \frac{\partial w}{\partial z}\right], \\
\frac{\partial p}{\partial y} & =\left[-\frac{\partial v}{\partial t}+v \frac{\partial^{2} v}{\partial y^{2}}+v \Delta_{s} v+F_{2}-u \frac{\partial v}{\partial x}-v \frac{\partial v}{\partial y}-w \frac{\partial v}{\partial z}\right]
\end{aligned}
$$

where the surface Laplacian is defined such that $\Delta_{s} \triangleq \partial^{2} / \partial x^{2}+\partial^{2} / \partial z^{2}$. Evaluating (2.2) at the wall, it follows that

$$
\begin{aligned}
& a_{2}=\frac{1}{v}\left[\frac{\partial a_{0}}{\partial t}+\frac{\partial d_{0}}{\partial x}-v \Delta_{s} a_{0}-\left.F_{1}\right|_{w}+a_{0} \frac{\partial a_{0}}{\partial x}+b_{0} a_{1}+c_{0} \frac{\partial a_{0}}{\partial z}\right], \\
& b_{2}=-\frac{\partial a_{1}}{\partial x}-\frac{\partial c_{1}}{\partial z}, \\
& c_{2}=\frac{1}{v}\left[\frac{\partial c_{0}}{\partial t}+\frac{\partial d_{0}}{\partial z}-v \Delta_{s} c_{0}-\left.F_{3}\right|_{w}+a_{0} \frac{\partial c_{0}}{\partial x}+b_{0} c_{1}+c_{0} \frac{\partial c_{0}}{\partial z}\right], \\
& d_{1}=\left[-\frac{\partial b_{0}}{\partial t}+v b_{2}+v \Delta_{s} b_{0}+\left.F_{2}\right|_{w}-a_{0} \frac{\partial b_{0}}{\partial x}-b_{0} b_{1}-c_{0} \frac{\partial b_{0}}{\partial z}\right] .
\end{aligned}
$$

Note that, to simplify the derivation, $d_{j}$ is computed after $b_{j+1}$. For all higher-order terms in the expansions of $u, v, w$, and $p$, general formulae may now be derived. With $j \geq 3$, we proceed further by taking $\partial^{j-2} / \partial y^{j-2}$ of (2.2), applying the binomial theorem to the derivatives of the nonlinear terms, and evaluating at the wall, which leads to:

$$
\begin{aligned}
& a_{j}=\frac{1}{v}\left[\frac{\partial a_{j-2}}{\partial t}+\frac{\partial d_{j-2}}{\partial x}-v \Delta_{s} a_{j-2}-\left.\frac{\partial^{j-2} F_{1}}{\partial y^{j-2}}\right|_{w}+\sum_{i=0}^{j-2}\left(\begin{array}{c}
j-2 \\
i
\end{array}\right)\left(a_{j-2-i} \frac{\partial a_{i}}{\partial x}+b_{j-2-i} a_{i+1}+c_{j-2-i} \frac{\partial a_{i}}{\partial z}\right)\right], \\
& b_{j}=-\frac{\partial a_{j-1}}{\partial x}-\frac{\partial c_{j-1}}{\partial z}, \\
& c_{j}=\frac{1}{v}\left[\frac{\partial c_{j-2}}{\partial t}+\frac{\partial d_{j-2}}{\partial z}-v \Delta_{s} c_{j-2}-\left.\frac{\partial^{j-2} F_{3}}{\partial y^{j-2}}\right|_{w}+\sum_{i=0}^{j-2}\left(\begin{array}{c}
j-2 \\
i
\end{array}\right)\left(a_{j-2-i} \frac{\partial c_{i}}{\partial x}+b_{j-2-i} c_{i+1}+c_{j-2-i} \frac{\partial c_{i}}{\partial z}\right)\right], \\
& d_{j-1}=\left[-\frac{\partial b_{j-2}}{\partial t}+v b_{j}+v \Delta_{s} b_{j-2}+\left.\frac{\partial^{j-2} F_{2}}{\partial y^{j-2}}\right|_{w}-\sum_{i=0}^{j-2}\left(\begin{array}{c}
j-2 \\
i
\end{array}\right)\left(a_{j-2-i} \frac{\partial b_{i}}{\partial x}+b_{j-2-i} b_{i+1}+c_{j-2-i} \frac{\partial b_{i}}{\partial z}\right)\right] .
\end{aligned}
$$

Combining this result with (2.1) and (2.3), it is seen that we may determine all terms in the Taylorseries expansions for $u, v, w$, and $p$ from the current values of the wall measurements of $\partial u / \partial y$, $\partial w / \partial y$, and $p$ and the derivatives of these quantities in $x, z$, and $t$, together with knowledge of the externally-applied momentum forcing and the velocity boundary conditions.

The Taylor-series expansions for the vorticity field follow directly from the Taylor-series expansions for the velocity field. Noting the definitions $\omega_{x}=\partial w / \partial y-\partial v / \partial z, \omega_{y}=\partial u / \partial z-\partial w / \partial x$, and $\omega_{z}=\partial v / \partial x-\partial u / \partial y$, inserting the Taylor-series expansions for the velocity and vorticity 
components, and matching like powers of $y$, it follows immediately for all $j$ that

$$
e_{j}=c_{j+1}-\frac{\partial b_{j}}{\partial z}, \quad f_{j}=\frac{\partial a_{j}}{\partial z}-\frac{\partial c_{j}}{\partial x}, \quad g_{j}=\frac{\partial b_{j}}{\partial x}-a_{j+1}
$$

\subsection{Taylor-series approximation: the case with homogeneous boundary conditions}

The expressions given above simplify greatly if we take $u_{w}=v_{w}=w_{w}=0$ and $F_{1}=P_{x}(t), F_{2}=$ $F_{3}=0$, as in the case of uncontrolled turbulent channel flow. Defining the notation

$$
D_{s}=\frac{\partial M_{1}}{\partial x}+\frac{\partial M_{3}}{\partial z}, \quad \mathcal{L}=\left(\frac{\partial}{\partial t}-v \Delta_{s}\right), \quad D_{d}=\frac{\partial M_{1}}{\partial x}-\frac{\partial M_{3}}{\partial z}, \quad R=\frac{\partial M_{3}}{\partial x}-\frac{\partial M_{1}}{\partial z},
$$

the first four nonzero terms in the expansions for the velocity, pressure, and vorticity can be written as

$$
\begin{aligned}
& u(y)=y M_{1}+\frac{y^{2}}{2 v}\left[\frac{\partial M_{2}}{\partial x}-P_{x}\right]+\frac{y^{3}}{6 v}\left[\mathcal{L} M_{1}-v \frac{\partial D_{s}}{\partial x}\right]+\frac{y^{4}}{24 v}\left[\mathcal{L} \frac{1}{v} \frac{\partial M_{2}}{\partial x}-\frac{1}{v} \dot{P}_{x}\right. \\
& \left.-\Delta_{s} \frac{\partial M_{2}}{\partial x}+M_{1} D_{d}+2 M_{3} \frac{\partial M_{1}}{\partial z}\right]+O\left(y^{5}\right) \\
& v(y)=-\frac{y^{2}}{2 v} v D_{s}-\frac{y^{3}}{6 v} \Delta_{s} M_{2}-\frac{y^{4}}{24 v}\left[\mathcal{L} D_{s}-v \Delta_{s} D_{s}\right]-\frac{y^{5}}{120 v}\left[\mathcal{L} \frac{1}{v} \Delta_{s} M_{2}-\Delta_{s} \Delta_{s} M_{2}\right. \\
& \left.+\frac{\partial M_{1} D_{s}}{\partial x}+\frac{\partial M_{3} D_{s}}{\partial z}-4\left(\frac{\partial M_{1}}{\partial x} \frac{\partial M_{3}}{\partial z}-\frac{\partial M_{3}}{\partial x} \frac{\partial M_{1}}{\partial z}\right)\right]+O\left(y^{6}\right), \\
& w(y)=y M_{3}+\frac{y^{2}}{2 v} \frac{\partial M_{2}}{\partial z}+\frac{y^{3}}{6 v}\left[\mathcal{L} M_{3}-v \frac{\partial D_{s}}{\partial z}\right]+\frac{y^{4}}{24 v}\left[\mathcal{L} \frac{1}{v} \frac{\partial M_{2}}{\partial z}-\Delta_{s} \frac{\partial M_{2}}{\partial z}\right. \\
& \left.-M_{3} D_{d}+2 M_{1} \frac{\partial M_{3}}{\partial x}\right]+O\left(y^{5}\right) \\
& p(y)=M_{2}-y v D_{s}-\frac{y^{2}}{2} \Delta_{s} M_{2}+\frac{y^{3}}{6} \mathrm{v} \Delta_{s} D_{s}+O\left(y^{4}\right), \\
& \omega_{x}(y)=M_{3}+\frac{y}{v} \frac{\partial M_{2}}{\partial z}+\frac{y^{2}}{2 v} \mathcal{L} M_{3}+\frac{y^{3}}{6 v}\left[\mathcal{L} \frac{1}{v} \frac{\partial M_{2}}{\partial z}-M_{3} D_{d}+2 M_{1} \frac{\partial M_{3}}{\partial x}\right]+O\left(y^{4}\right), \\
& \omega_{y}(y)=-y R-\frac{y^{3}}{6 v} \mathcal{L} R+\frac{y^{4}}{24 v}\left[M_{3} \Delta_{s} M_{1}-M_{1} \Delta_{s} M_{3}-\frac{\partial M_{1} R}{\partial x}-\frac{\partial M_{3} R}{\partial z}\right]+O\left(y^{5}\right), \\
& \omega_{z}(y)=-M_{1}-\frac{y}{v} \frac{\partial M_{2}}{\partial x}-\frac{y^{2}}{2 v} \mathcal{L} M_{1}-\frac{y^{3}}{6 v}\left[\mathcal{L} \frac{1}{v} \frac{\partial M_{2}}{\partial x}+M_{1} D_{d}+2 M_{3} \frac{\partial M_{1}}{\partial z}\right]+O\left(y^{4}\right) .
\end{aligned}
$$

Note that all of these Taylor series reconstruct the flow state by extrapolation of the local values of the flow measurements and the external forcing and their derivatives in space and time.

\subsection{The linear case - pressure measurements not required}

Consider now an unsteady Stokes flow in a channel, governed by the Navier-Stokes equation (1.1) with all nonlinear terms removed. This system requires three boundary conditions on the walls in 
order to be well posed in the sense of solving the evolution of the flow forward in time from known initial conditions. Let us now look at the details of the Taylor-series expansions to determine how much information on the walls is sufficient in order to uniquely determine the unsteady flow state everywhere in the channel flow domain at time $t$ without knowledge of the initial conditions via measurements at the walls.

1) If one looks at the wall quantities at time $t$, one needs an infinite number of quantities (boundary conditions and stress and their derivatives in time up to infinite order) to reconstruct the Taylor series.

2) If one looks at the wall quantities in a neighborhood of time $t$, one needs five quantities on the walls (boundary conditions and streamwise and spanwise wall skin friction; wall pressure measurements are not required). From these five wall quantities in a neighborhood of time $t$, the entire Taylor-series expansion may be determined. This is because, in this case, the sixth wall quantity (wall pressure) can be reproduced from the other five wall quantities in a neighborhood of time $t$ via solution of an (elliptic ) 3D Poisson equation with Neumann boundary conditions:

$$
\left(\frac{\partial^{2}}{\partial x^{2}}+\frac{\partial^{2}}{\partial y^{2}}+\frac{\partial^{2}}{\partial z^{2}}\right) p=\frac{\partial F_{1}}{\partial x}+\frac{\partial F_{1}}{\partial y}+\frac{\partial F_{1}}{\partial z} \quad \text { with }\left.\quad \frac{\partial p}{\partial n}\right|_{w}=d_{1}
$$

where $d_{1}$ may be determined from (2.3) and (2.1) without wall pressure measurements. Note that in order to perform a local reconstruction of the flow, we need either

a) local information of the nine quantities $\left\{u_{w}, v_{w}, w_{w}, F_{1}, F_{2}, F_{3},\left.\frac{\partial u}{\partial y}\right|_{w},\left.p\right|_{w},\left.\frac{\partial w}{\partial y}\right|_{w}\right\}$ and their space and time derivatives, or

b) the five quantities $\left\{u_{w}, v_{w}, w_{w},\left.\frac{\partial u}{\partial y}\right|_{w},\left.\frac{\partial w}{\partial y}\right|_{w}\right\}$ and their time derivatives everywhere on the walls and the three quantities $\left\{F_{1}, F_{2}, F_{3}\right\}$ and their time derivatives everywhere in the channel.

\subsection{The nonlinear case - pressure measurements required}

When moving from the linear case to the nonlinear case, the pressure can no longer be determined from a 3D Poisson equation based on $\left\{u_{w}, v_{w}, w_{w}, F_{1}, F_{2}, F_{3},\left.M_{1} \triangleq \frac{\partial u}{\partial y}\right|_{w},\left.M_{3} \triangleq \frac{\partial w}{\partial y}\right|_{w}\right\}$ alone, and thus strategy b described above (without wall pressure measurements) is no longer viable. Note also that, via simple combination of (1.1) and (1.2), it is possible to write a 2D Poisson equation for the wall pressure; however, it is not possible solve this equation for $\left.p\right|_{w}$ based on $\left\{u_{w}, v_{w}, w_{w}, F_{1}, F_{2}, F_{3}, M_{1}, M_{3}\right\}$ alone.

The wall pressure measurement $\left.M_{2} \triangleq p\right|_{w}$ plays an important role in the higher-order terms in the Taylor-series expansions derived above; without it, these expansions must be truncated at very low order. Thus, though pressure measurements may be dispensed with in the global flow reconstruction problem in the linear setting (replacing the wall pressure measurements with the solution of an elliptic Poisson problem), the derivation presented above indicates a valuable role for wall pressure measurements in the global reconstruction of the state of the nonlinear turbulent channel flow system, regardless of the technique actually used to assimilate these measurements into an 
estimate of the state of the turbulent flow.

\subsection{Evaluation of truncated Taylor series in a DNS of turbulent channel flow}

We now investigate the range of validity of the Taylor-series expansions computed in $\$ 2.2$ subject to various levels of truncation. For this purpose, we use a DNS database for an uncontrolled, constant-mass flux turbulent channel flow at $R e_{\tau}=180$ using the spectral/finite-difference/spectral code of Bewley, Moin, \& Temam (2001) on a $256 \times 129 \times 256$ numerical grid. Using the wall information (i.e., the measurements $M_{1}, M_{2}$, and $M_{3}$ ) to evaluate the coefficients in the expansions listed in $\$ 2.2$ (truncated after the $i$ 'th-order term), we can reconstruct the velocity and vorticity components and the pressure. The quality of the reconstruction (as a function of the level of truncation, $i$, and the distance from the wall, $y$ ) may be characterized by the correlation of the perturbation components of the reconstructed and actual fields, given by

$$
\operatorname{Corr}_{y}\left(q_{r e c}^{\prime}, q_{a c t}^{\prime}\right) \triangleq \frac{\int_{0}^{L_{1}} \int_{0}^{L_{3}} q_{r e c}^{\prime}(y) q_{a c t}^{\prime}(y) d x d z}{\sqrt{\int_{0}^{L_{1}} \int_{0}^{L_{3}}\left(q_{r e c}^{\prime}(y)\right)^{2} d x d z} \sqrt{\int_{0}^{L_{1}} \int_{0}^{L_{3}}\left(q_{a c t}^{\prime}(y)\right)^{2} d x d z}}
$$

or via the corresponding planewise error norm, given by

$$
\operatorname{Errn}_{y}\left(q_{r e c}^{\prime}, q_{a c t}^{\prime}\right) \triangleq \frac{\sqrt{\int_{0}^{L_{1}} \int_{0}^{L_{3}}\left(q_{r e c}^{\prime}(y)-q_{a c t}^{\prime}(y)\right)^{2} d x d z}}{\sqrt{\int_{0}^{L_{1}} \int_{0}^{L_{3}}\left(q_{a c t}^{\prime}(y)\right)^{2} d x d z}},
$$

where $q^{\prime}$ denotes the perturbation component (with the mean components subtracted off) of any quantity chosen from the set $\left\{u, v, w, p, \omega_{x}, \omega_{y}, \omega_{z}\right\}$, and the subscripts rec and act correspond to the reconstructed and actual fields respectively. The correlations and planewise error norms are computed for the perturbation fields to avoid the bias that might be introduced by the mean field. Thus, the statistics at a given distance $y$ from the wall are computed by averaging the instantaneous perturbation fields over the streamwise and spanwise directions; upon discretization, this corresponds to averaging over $2^{16}$ grid points for each datapoint reported. Spatial differentiation of the wall measurements (in the directions $x$ and $z$ ) was carried out spectrally, and temporal differentiation was carried out using a second-order central-difference approximation. In Figures 1 and 2, we show the dependence of the correlation (2.4) and the planewise error norm (2.5), respectively, for all the quantities in the set $\left\{u, v, w, p, \omega_{x}, \omega_{y}, \omega_{z}\right\}$ as a function of the distance from the wall $y$ and the order of truncation $i$. The wall-normal coordinate is given in wall units as $y^{+}=y /\left(\mathrm{v} / u_{\tau}\right)$,

where $u_{\tau}=\sqrt{\tau_{w} / \rho}$ and $\tau_{w}$ is the average skin friction on the wall. In all cases we note a systematic improvement of the reconstruction as more terms are included in expansion.

An alternative representation of the convergence of the Taylor-series expansions listed in $\S 2.2$ as the number of terms is increased is presented in Figure 3. This figure shows the joint probability density function (JPDF) of the truncated Taylor-series approximation of the wall-normal velocity with the actual wall-normal velocity evaluated at $y^{+}=3$ as the number of terms in the truncated Taylor series is increased. The rapid approach of the JPDF towards a diagonal line indicates the convergence of the Taylor-series expansion. Figure 4 repeats the calculation of Figure 3, but eval- 

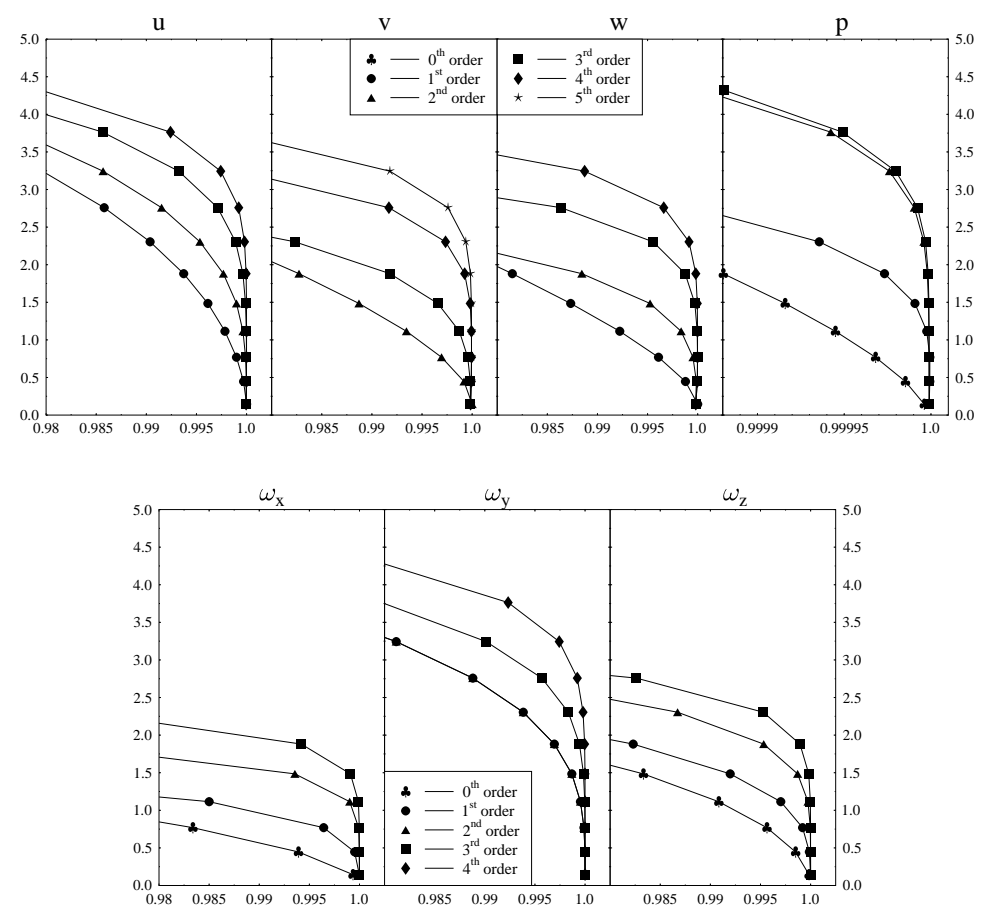

Fig. 1. Correlations of the components of the reconstructed and actual velocity, pressure, and vorticity fields as a function of the distance from the wall, $y^{+}$, in a turbulent channel flow at $R e_{\tau}=180$. Reconstructions were computed by retaining the terms indicated in the Taylor-series expansions listed in $\$ 2.2$, and the correlations were computed according to (2.4).
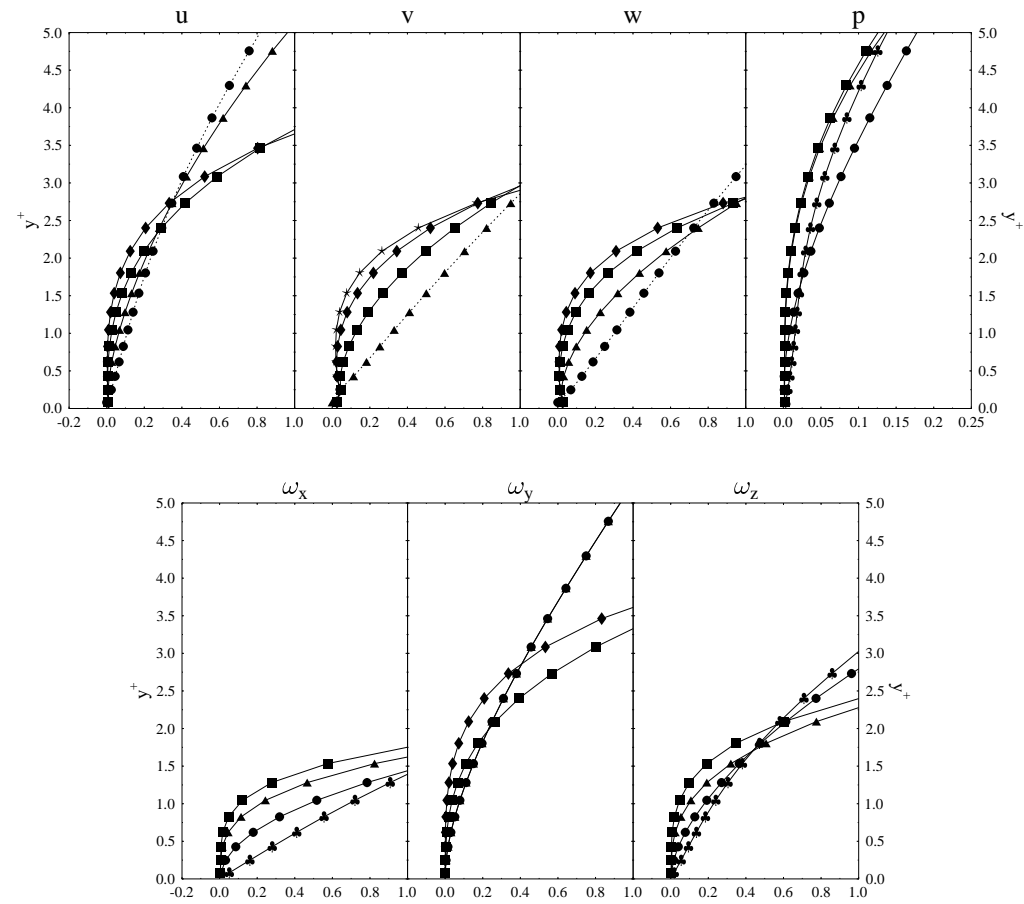

Fig. 2. Error norms corresponding to the correlations plotted in Figure 2. 

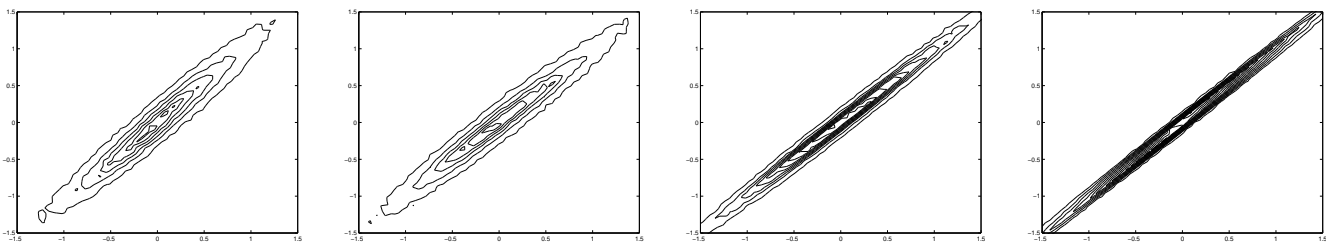

Fig. 3. Joint probability density functions (JPDF) of the truncated Taylor-series approximations of the $v$-component of the flow (vertical axis) with the actual flow (horizontal axis) evaluated at $y^{+}=3$, using (from left to right) truncation of the Taylor series after 2, 3, 4, and 5 terms.
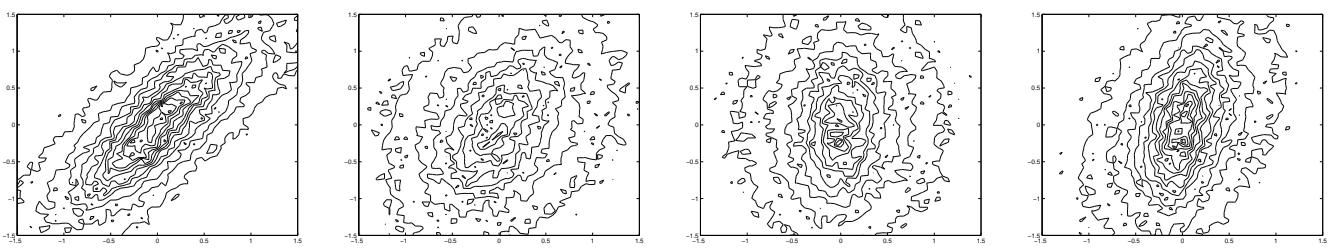

Fig. 4. The same as Figure 2 but evaluated at $y^{+}=10$. Note that the subfigure on the left closely approximates that reported in Figure 6c of Choi, Moin, \& Kim (1994).
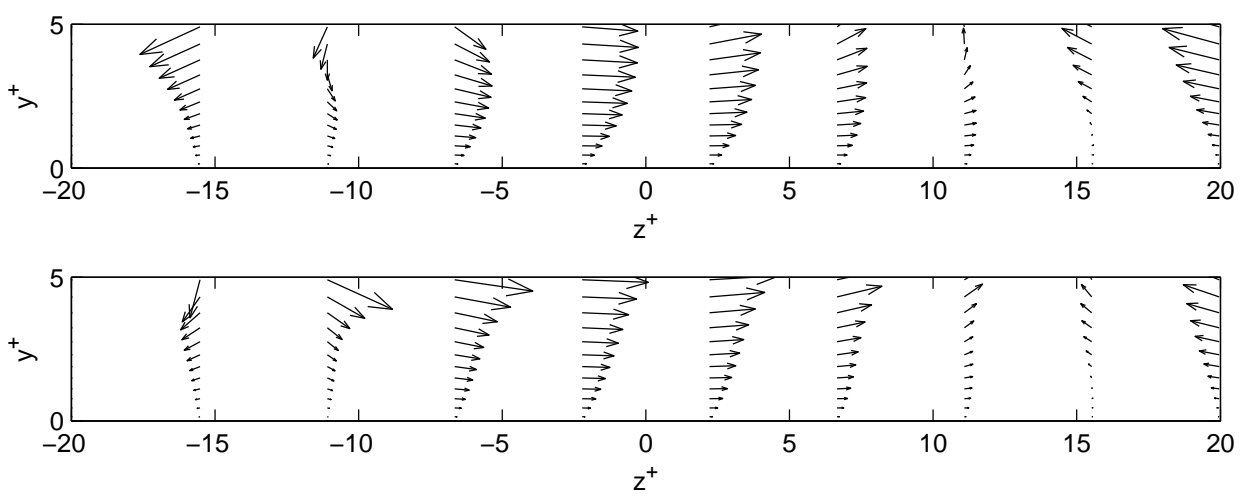

Fig. 5. The actual (top) and reconstructed (bottom) turbulent flow fields in the cross-flow plane near the wall, using 4 nonzero terms in the Taylor series reconstruction. Note that the accuracy of the state estimate is degraded away from the wall, but the velocity fields are in general agreement close to the wall. Note in particular that the sweep event centered at $z^{+}=-10$ in the actual flow appears to be centered at $z^{+}=-15$ in the 4-term reconstruction of the flow.

uated at $y^{+}=10$. Even when including several terms of the Taylor-series expansion, the JPDF resembles a shotgun blast. From the terms which are evaluated here, convergence of the Taylor series can not be detected. Carrying the expansion to higher orders is not feasible due to the limited accuracy of the numerical database.

Figure 5 illustrates flow visualizations in the cross-flow plane of the actual flow and its Taylor series reconstruction based on skin-friction and pressure measurements on the wall, again indicating the convergence of the Taylor series near the wall.

The main messages to be taken from Figures 1-5 are: 
A. The higher-order information available when both wall skin friction and wall pressure are used as measured quantities is quite significant in the static reconstruction of the flow adjacent to the wall by extrapolation of the measured quantities.

B. However, the radius of convergence of Taylor-series expansions of the flow evaluated at the wall is relatively small (less than 10 wall units).

Note that point B does not invalidate point $\mathrm{A}$.

\subsection{Analyticity and unique continuation}

Mathematical proof of the space analyticity of solutions of Navier-Stokes systems on the attractor of fully-developed turbulence in infinite and periodic domains (with sufficiently-smooth forcing) is well established (see Grujić \& Kukavica 1998). Extension of this proof to establish the space analyticity of fully-developed channel-flow turbulence is straightforward (I. Kukavica and M. Ziane, private communication), and will be reported separately. The unique continuation theorem (see, e.g., Saut \& Scheurer 1987) implies that there is a unique analytic function in an entire channelflow domain which coincides exactly with the analytic function given by a (converged) Taylorseries expansion in the vicinity of the wall (e.g., on the domain $0<y^{+}<3$ ). It is thus (in theory) possible to reconstruct the entire (analytic) solution of the flow in the channel based on complete Taylor series information which is convergent only in the immediate vicinity of the wall. Formally, an algorithm to reconstruct the entire flow solution might then proceed as follows:

1) Based on the Taylor series expansion of $u$ on the wall, compute $u, \partial u / \partial y, \partial^{2} u / \partial y^{2}$, etc., on some plane near the wall (say, $y^{+}=3$ ).

2) Based on the information computed in step 1, compute a new Taylor series expansion about the plane $y^{+}=3$ and evaluate it to determine $u, \partial u / \partial y, \partial^{2} u / \partial y^{2}$, etc., at $y^{+}=6$.

3) Based on the information computed in step 2, compute $u$ and its derivatives at $y^{+}=9$. Continue marching in this fashion, one plane at a time, to reconstruct the flow solution in the entire channel.

Unfortunately, the proof of the convergence of this algorithm is only formal, as it requires the exact convergence of all of the calculations in step 1 before proceeding to step 2 . If these series expansions are truncated, errors accumulate, and the algorithm listed above breaks down. Thus, this algorithm can not be used to extend the domain of convergence of the original Taylor series expressed on the wall in a numerical calculation which retains only a finite number of terms. More practical algorithms to reconstruct the flow solution based on the analyticity of Navier-Stokes solutions coupled with accurate truncated Taylor-series expansions on the wall will be explored in future work.

However, this argument is sufficient to establish that wall measurements of streamwise and spanwise skin friction and pressure in a neighborhood of time $t$ combine to provide a unique "footprint" of a turbulent flow state; no other fully-developed turbulent flow realization (subject to the same externally-applied forcing) can possibly have the same footprint, regardless of its initial conditions. 
The same can not be said if one of the three measurements is missing.

\subsection{Extrapolation with global basis functions}

The above extrapolations with Taylor series represent an lower-triangular relationship between the vector containing all of the coefficients of the Taylor-series expansions (truncated at some order) and the vector containing all of the measurements and their time derivatives (also truncated at the appropriate order). In other words, when additional information concerning higher-order time derivatives of the measurements is provided, additional higher-order terms in the Taylor series may be determined, but the lower-order terms in the Taylor series remain unchanged.

In the spirit of the LSE approach mentioned in the introduction, the velocity field may be expanded into global basis functions, such as Fourier in $x$ and $z$ and Chebyshev in $y$. However, looking at a single Fourier mode, the relationship between the vector containing all of the coefficients of the Chebyshev expansions (truncated at some order) and the vector containing all of the measurements and their time derivatives (also truncated at the appropriate order) found via the equations of $\S 2.1$ turns out to be upper-triangular. In other words, when information concerning higher-order time derivatives of the measurements is provided, all terms in the series expansion are modified. For this reason, this type of expansion did not yield coefficients which converged quickly in the present investigation. Expansions based on POD modes are similarly ill-behaved; the matrices involved with these expansions at each Fourier mode are full. For this reason, expansions into series with global basis functions was not pursued further in the present work.

\section{Approximate state estimation from noisy wall measurements}

The above results highlight the fundamental importance of using all three flow quantities available at the wall when attempting to reconstruct a flow in the hypothetical case in which perfect measurements are available on the wall in a neighborhood of time $t$.

We now address the relation of the above findings on the hypothetical problem of exact state reconstruction with precise wall information to the practical problem of approximate state estimation with noisy measurements at the wall. Such a problem is often referred to as "variational data assimilation" or "4D-var", and plays a central role in the field of numerical weather prediction (for a recent review of this active field of research, see, e.g., Li, Navon, \& Zhu 2000). There are essentially two model-based approaches to the problem of state estimation in this setting: adjoint-based strategies and Riccati-based strategies, the latter of which are often based on reduced-rank extended Kalman filters. Complete description of these two approaches is beyond the scope of the present paper. However, in light of the observations made previously concerning the valuable role of wallpressure measurements in the problem of exact state reconstruction near the wall in wall-bounded turbulent flows (that is, in the nonlinear setting), it is enlightening to review the formulation for adjoint-based state estimation with noisy measurements at the wall. In the present section, rather than taking the wall at $y=0$, we switch to an $x_{1}-x_{2}-x_{3}$ coordinate system, and consider an en- 
tire channel-flow system in the domain $\left(0 \times L_{1}\right) \times(-1 \times 1) \times\left(0 \times L_{3}\right)$. For simplicity and without further mention, we consider periodic boundary conditions in $x_{1}$ and $x_{3}$ on all field variables in the derivation that follows.

Define first an (unknown) noise vector $\mathbf{w}=\left(w_{1} w_{2} w_{3}\right)^{T}$ and a noisy wall measurement vector $\mathbf{m}=\left(m_{1} m_{2} m_{3}\right)^{T}$, where $\left.m_{1} \triangleq \frac{\partial u_{1}}{\partial \bar{n}}\right|_{w}+w_{1},\left.m_{2} \triangleq p\right|_{w}+w_{2}$, and $\left.m_{3} \triangleq \frac{\partial u_{3}}{\partial \bar{n}}\right|_{w}+w_{3}$, distributed in time over an "assimilation window" $[0, T]$ and in space over the channel walls for an "actual" channel-flow system. For convenience, $\overline{\mathbf{n}}$ is defined as an inward-facing normal. We now seek to determine the (unknown) initial state $\Phi$ of a model system everywhere inside the channel such that, when advanced in time over the interval $0 \rightarrow T$, the model reproduces the observed measurements to the maximum extent possible. Defining the state vector $\mathbf{q}$, the perturbation vector $\mathbf{q}^{\prime}$, and the adjoint vector $\mathbf{q}^{*}$ such that $\mathbf{q}=\left(\begin{array}{l}\mathbf{u} \\ p\end{array}\right), \mathbf{q}^{\prime}=\left(\begin{array}{l}\mathbf{u}^{\prime} \\ p^{\prime}\end{array}\right), \mathbf{q}^{*}=\left(\begin{array}{l}\mathbf{u}^{*} \\ p^{*}\end{array}\right)$, we first write the Navier-Stokes equation (1.1) governing the model system in the compact form

$$
\begin{aligned}
& \mathcal{N}(\mathbf{q})=\left(\begin{array}{c}
\frac{\partial \mathbf{u}}{\partial t}+(\mathbf{u} \cdot \nabla) \mathbf{u}+\nabla p-v \Delta \mathbf{u} \\
\nabla \cdot \mathbf{u}
\end{array}\right)=\left(\begin{array}{c}
P_{x} \mathbf{i} \\
0
\end{array}\right) \quad \text { in } \Omega \times(0, T) \\
& \text { with }\left.\mathbf{u}\right|_{t=0}=\Phi,\left.\quad \mathbf{u}\right|_{w}=0 .
\end{aligned}
$$

The objective in the present optimization problem is defined mathematically as the minimization over all feasible initial conditions $\Phi$ of a cost functional $\mathcal{I}(\Phi)$ which represents the "misfit" of the measurements in the actual and reconstructed systems

$$
\mathcal{I}(\Phi)=\frac{1}{2} \int_{0}^{T}\left[\ell_{1}\left\|\frac{\partial u_{1}}{\partial^{-} n}-m_{1}\right\|_{w}^{2}+\ell_{2}\left\|p-m_{2}\right\|_{w}^{2}+\ell_{3}\left\|\frac{\partial u_{3}}{\partial^{-} n}-m_{3}\right\|_{w}^{2}\right] d t
$$

where the coefficients $\ell_{1}, \ell_{2}, \ell_{3}$, and the norm $\|\cdot\|_{w}$ are defined appropriately to measure the deviation of the model system from the measurements of the actual flow on the channel walls at $x_{2}= \pm 1$ (denoted here by $w$ ). Note that $\ell_{2}$ is proportional to the square of the (constant) fluid density, $\rho^{2}$, and $\ell_{1}$ and $\ell_{3}$ are proportional to the square of the (constant) fluid viscosity, $\mu^{2}$, in order to make (3.2) dimensionally consistent. In the present work we will use $L_{2}$ norms such that $\|f\|_{w}^{2} \triangleq \int_{w} f^{2} d S$. The initial conditions $\Phi$ which minimize $\mathcal{I}(\Phi)$ may be found by a gradient-based search. To identify the gradient, an inner product over $\Omega$ must first be defined; in the present work, we will use $L_{2}$ inner products such that $\langle\mathbf{f}, \mathbf{g}\rangle_{\Omega} \triangleq \int_{\Omega} \mathbf{f} \cdot \mathbf{g} d V$. The functional gradient $\mathcal{D} \mathcal{g} / \mathcal{D} \Phi$ is then defined such that, for $\varepsilon \ll 1$ and for any feasible $\Phi^{\prime}$,

$$
\begin{aligned}
& \mathcal{I}\left(\Phi+\varepsilon \Phi^{\prime}\right) \approx \mathcal{J}(\Phi)+\varepsilon\left\langle\frac{\mathcal{D} \mathcal{I}}{\mathcal{D} \Phi}, \Phi^{\prime}\right\rangle_{\Omega}=\mathcal{H}(\Phi)+\varepsilon \int_{\Omega} \frac{\mathcal{D} \mathcal{J}}{\mathcal{D} \Phi} \cdot \Phi^{\prime} d V \\
& =\mathcal{I}(\Phi)+\varepsilon \int_{0}^{T} \int_{w}\left[\ell_{1}\left(\frac{\partial u_{1}}{\partial^{-} n}-m_{1}\right) \frac{\partial u_{1}^{\prime}}{\partial^{-} n}+\ell_{2}\left(p-m_{2}\right) p^{\prime}+\ell_{3}\left(\frac{\partial u_{3}}{\partial^{-} n}-m_{3}\right) \frac{\partial u_{3}^{\prime}}{\partial^{-} n}\right] d S d t
\end{aligned}
$$

where the equation governing $\mathbf{q}^{\prime}$ is found by inserting $\Phi+\varepsilon \Phi^{\prime}$ for $\Phi$ and $\mathbf{q}+\varepsilon \mathbf{q}^{\prime}$ for $\mathbf{q}$ in (3.1) and 
assuming $\varepsilon \ll 1$; collecting the terms proportional to $\varepsilon$, this results in

$$
\begin{gathered}
\mathcal{L} \mathbf{q}^{\prime}=\left(\begin{array}{c}
\frac{\partial \mathbf{u}^{\prime}}{\partial t}+(\mathbf{u} \cdot \nabla) \mathbf{u}^{\prime}+\left(\mathbf{u}^{\prime} \cdot \nabla\right) \mathbf{u}+\nabla p^{\prime}-v \Delta \mathbf{u}^{\prime} \\
\nabla \cdot \mathbf{u}^{\prime}
\end{array}\right)=0 \quad \text { in } \Omega \times(0, T), \\
\quad \text { with }\left.\quad \mathbf{u}^{\prime}\right|_{t=0}=\Phi^{\prime},\left.\quad \mathbf{u}^{\prime}\right|_{w}=0
\end{gathered}
$$

Note that (3.4) reflects a linear relationship between $\mathbf{q}^{\prime}$ and $\Phi^{\prime}$, though this linear relationship is not yet expressed in a convenient form from which the functional gradient $\mathcal{D} \mathcal{J} / \mathcal{D} \Phi$ in (3.3) may be identified. Towards this end, we perform an adjoint analysis. Defining first a duality pairing (in the present work, we will use the $L_{2}$ duality pairing $\left.\langle\mathbf{f}, \mathbf{g}\rangle_{\Omega \times(0, T)} \triangleq \int_{0}^{T} \int_{\Omega} \mathbf{f} \cdot \mathbf{g} d S d t\right)$, straightforward integration by parts (see, e.g., Bewley, Moin, \& Temam 2001) leads to an identity of the form

$$
\left\langle\mathbf{q}^{*}, \mathcal{L} \mathbf{q}^{\prime}\right\rangle_{\Omega \times(0, T)}=\left\langle\mathcal{L}^{*} \mathbf{q}^{*}, \mathbf{q}^{\prime}\right\rangle_{\Omega \times(0, T)}+b,
$$

where

$$
\begin{aligned}
& \mathcal{L}^{*} \mathbf{q}^{*}=\left(\begin{array}{c}
-\frac{\partial \mathbf{u}^{*}}{\partial t}-\mathbf{u} \cdot\left[\nabla \mathbf{u}^{*}+\left(\nabla \mathbf{u}^{*}\right)^{T}\right]-\nabla p^{*}-v \Delta \mathbf{u}^{*}, \\
-\nabla \cdot \mathbf{u}^{*}
\end{array}\right), \\
& b=\left.\int_{\Omega}\left(u_{j}^{*} u_{j}^{\prime}\right)\right|_{t=0} ^{t=T} d \mathbf{x}-\int_{0}^{T} \int_{w} \eta\left[p^{*} u_{j}^{\prime}+u_{j}^{*} p^{\prime}+u_{i}^{*}\left(u_{j} u_{i}^{\prime}+u_{j}^{\prime} u_{i}\right)-v\left(u_{i}^{*} \frac{\partial u_{i}^{\prime}}{\partial x_{j}}-u_{i}^{\prime} \frac{\partial u_{i}^{*}}{\partial x_{j}}\right)\right] d \mathbf{x} d t .
\end{aligned}
$$

Leveraging this identity, consider now an adjoint state $\mathbf{q}^{*}$ defined via the equation

$$
\begin{aligned}
& \mathcal{L}^{*} \mathbf{q}^{*}=0 \text { in } \Omega \times(0, T), \quad \text { with }\left.\quad \mathbf{u}^{*}\right|_{t=T}=0 \\
&\left.u_{1}^{*}\right|_{w}=\ell_{1} \frac{1}{v}\left(\frac{\partial u_{1}}{\partial^{-} n}-m_{1}\right),\left.\quad u_{2}^{*}\right|_{w}=\ell_{2}{ }^{-} n\left(p-m_{2}\right),\left.\quad u_{3}^{*}\right|_{w}=\ell_{3} \frac{1}{v}\left(\frac{\partial u_{3}}{\partial^{-} n}-m_{3}\right) .
\end{aligned}
$$

Note that the difficulty involved with numerically solving the adjoint system given above via a backward march from $t=T$ to $t=0$ is almost the same as the difficulty involved with solving the original system (3.1). The identity (3.5) may be used to put all of the pieces together: inserting the perturbation equation (3.4) and the adjoint equation (3.6) into the identity (3.5) and simplifying, the perturbation of the cost functional given in (3.3) may be rewritten in the convenient form

$$
\int_{\Omega} \frac{\mathcal{D} \mathcal{I}}{\mathcal{D} \Phi} \cdot \Phi^{\prime} d V=\left.\int_{\Omega} \mathbf{u}^{*}\right|_{t=0} \cdot \Phi^{\prime} d V
$$

As this derivation is valid for all $\Phi^{\prime}$, we may identify the functional gradient which we seek

$$
\frac{\mathcal{D} \mathcal{I}}{\mathcal{D} \Phi}=\left.\mathbf{u}^{*}\right|_{t=0} .
$$

Physically, the adjoint field evaluated in the domain where the control is defined represents the sensitivity of the cost functional (3.2) to modification of the control variable. In the present problem, this "control variable" is simply the unknown initial condition. 
Estimation of the entire state of a turbulent channel flow based on wall measurements alone is an extremely challenging task, as the turbulent channel flow system is governed by a nonlinear PDE exhibiting complex multiscale dynamics which are high dimensional and rapidly evolving. Figure 6 illustrates the best results we have obtained so far on this challenging problem at $R e_{\tau}=100$, using the algorithm derived above, after 40 iterations of the adjoint-based optimization, taking the optimization interval $T$ to be a relatively short 100 viscous time units, and using a very bad initial guess in the estimator (simply the mean flow). To perform this calculation, a "truth model" (that is, a DNS of a turbulent flow in a channel) was first computed. Based solely on the wall measurements from this "actual flow", a state estimate was optimized using the adjoint algorithm described above. The accuracy of this state estimate (at the center of the optimization interval $[0, T]$ ), as shown in Figure 6, is fairly good near the wall but degraded near the center of the domain, as expected. The adjoint-based state estimation algorithm described above may be significantly refined by selecting other norms, duality pairings and inner products besides the simple $L_{2}$ forms used in the above discussion. This matter is discussed thoroughly in Protas, Bewley, \& Hagen (2003), and will be explored thoroughly in the context of the present estimation problem in future work.

The primary purpose of presenting this derivation in this paper is to illustrate that there are exactly three possibilities for forcing the relevant adjoint equation on the boundary, as shown in (3.6). The misfits of the three measurements $m_{1}, m_{2}$, and $m_{3}$ exhaust all possibilities for the forcing of this adjoint problem from the wall. Moreover, given the linearity of the adjoint system with respect to the boundary conditions, the gradient information obtained via the misfits of the three different types of measurements in this problem is linearly additive. As seen in Figure 6, the resulting wall-normal velocity and pressure reconstructions in the flow are markedly improved when wall-pressure measurements are used in the adjoint-based state estimation algorithm.

\section{Discussion}

Significant progress has been made in recent years in the area of boundary control of turbulent flow systems using model-based control theory and complete state information [see, e.g., Bewley (2001) for a recent review]. However, the dual problem of estimation of the flow state based on boundary measurements of the turbulent flow system essentially remains open. Both problems must be solved if model-based control of turbulent flow systems is to become engineering reality.

The present paper shows a strategy for determining a turbulent channel flow at time $t$ based solely on measurements of wall skin friction and pressure available in a neighborhood of time $t$, without the knowledge of the initial conditions at some time $t_{0}<t$. Numerical computations show that the resulting algorithm based on Taylor series expansions converges up to a few viscous units from the wall. As an alternative, a variational adjoint-based state estimation algorithm was presented, which was shown to lead to a far better reconstruction of the flow in the whole domain. Analysis of both of these approaches emphasizes the valuable role that pressure measurements play in the reconstruction of nonlinear turbulent flows. This is contrasted with linear (Stokes) problems, where the pressure measurements can be dispensed with without affecting the reconstruction. 

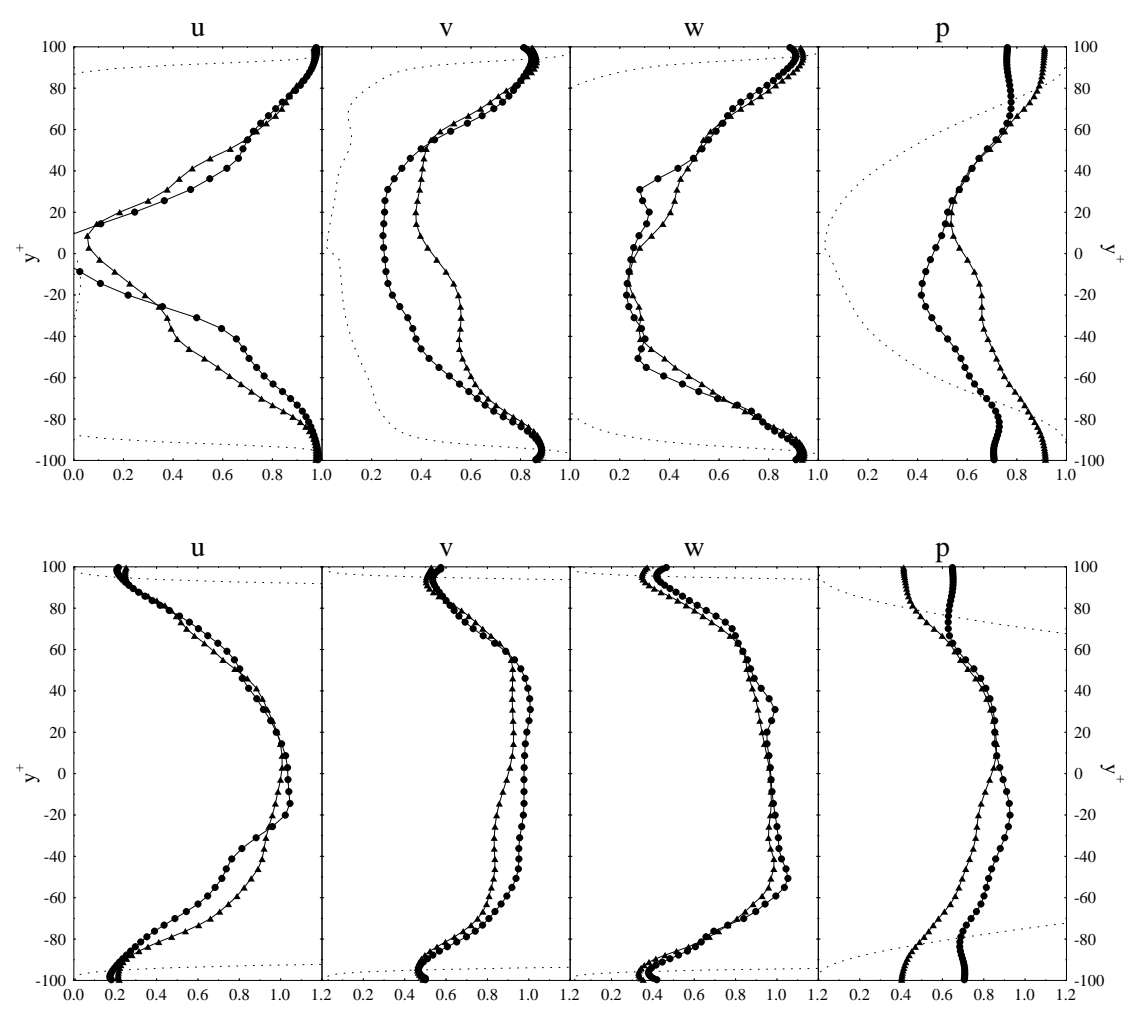

Fig. 6. Correlations of the actual and reconstructed quantities (top) and the corresponding planewise norms of the state estimation error (bottom) determined using the adjoint-based state estimation approach; $(\Delta$ ) adjoint-based reconstruction using both skin friction and wall-pressure measurements, $(\bullet)$ adjoint-based reconstruction using skin friction measurements alone, (........) Taylor-series extrapolation using the first 4 nonzero terms and both skin friction and wall-pressure measurements. Note that the adjoint-based approach yields significantly better results farther from the walls; cf. Figures 1 and 2 for lower-order Taylor-series extrapolations in the immediate vicinity of the wall.

\section{Acknowledgements}

This article is dedicated to the memory of Uwe Dallmann, whose characterizations of the "footprints" of separated flows (see, e.g., Dallmann \& Schewe 1987 and Rütten \& Dallmann 2001) inspired the present investigation. The authors thank Mohammed Ziane and Igor Kukavica for several interesting discussions related to this work.

\section{References}

[1] AdRian, R.J. 1977 On the Role of Conditional Averages in Turbulence Theory. In Turbulence in Liquids, (eds. J. Zakin and G. Patterson), Science Press, Princeton, 323-332.

[2] Balogh, A., LiU, W.-J., \& Krstic, M. 2001 Stability enhancement by boundary control in 2-D channel flow. IEEE Trans. Aut. Control 46, 1696-1711. 
[3] Bewley, T.R. 2001 Flow control: new challenges for a new Renaissance. Progress in Aerospace Sciences 37, 21-58.

[4] Bewley, T.R., Moin, P., \& Temam, R. 2001 DNS-based predictive control of turbulence: an optimal benchmark for feedback algorithms. J. Fluid Mech. 447, 179-225.

[5] Choi, H., Moin, P., \& Kim, J. 1994 Active turbulence control for drag reduction in wall-bounded flows. J. Fluid Mech. 262, 75-110.

[6] Cole, D.R., Glauser, M.N., \& Guezennec, Y.G. 1992 An Application of Stochastic Estimation to the Jet Mixing Layer. Phys. Fluids A 4, 192.

[7] Dallmann, U., \& Schewe, G. 1987 On topological changes of separating flow structures at transition Reynolds numbers. AIAA-87-1266.

[8] GAD-EL-HAK, M. 2001 Flow control: passive, active, and reactive flow management. Cambridge.

[9] Grujić, Z., \& KuKAVICA, I. 1998 Space analyticity for the Navier-Stokes and related equations with initial data in $L^{p}$. J. Funct. Anal., 152, 447-466.

[10] GunZBurger, M.D. 2002 Perspectives in flow control and optimization. SIAM.

[11] Gunzburger, M.D., \& LeE, H.C. 1996 Feedback control of Karman vortex shedding. J. Appl. Mech., 63, 828-835.

[12] Hernandez, R.H., Baudet, C., \& Fauve, S. 2000 Controlling the Benard-von Karman instability in the wake of a cylinder by driving the pressure at the front stagnation point. European Physical Journal $B, 14,773-781$.

[13] Johansson, A.V., Her, J.-Y., \& Haritonidis, J.H. 1987 On the generation of high-amplitude wall-pressure peaks in turbulent boundary layers and spots. J. Fluid Mech., 175, 119-142.

[14] Kim, J., ChOI, J.-I., \& Sung, H.J. 2002 Relationship between wall pressure fluctuations and streamwise vortices in a turbulent boundary layer. Phys. Fluids, 14, 898-901.

[15] Koumoutsakos, P. 1999 Vorticity flux control for a turbulent channel flow. Phys. Fluids, 11, 248250.

[16] Kravchenko, A.G., Choi, H., \& Moin, P. 1993 On the relation of near-wall streamwise vortices to wall skin friction in turbulent boundary layers. Phys. Fluids A, 5, 3307-3309.

[17] LEE, I., \& SUNG, H.J. 2002 Multiple-arrayed pressure measurement for investigation of the unsteady flow structure of a reattaching shear layer. J. Fluid Mech., 463, 377-402.

[18] LI, Z.J., NAvon, I.M., \& ZHU, Y.Q. 2000 Performance of 4D-Var with different strategies for the use of adjoint physics with the FSU global spectral model. Monthly Weather Review 128, 668-688.

[19] Luchini, P., Bottaro, A., \& Zuccher, S. 2001 Optimal perturbations and control of nonlinear boundary layer. Bulletin of The American Physical Society, 46 (10), 184.

[20] Podvin, B., \& Lumley, J. 1998 Reconstructing the flow in the wall region from wall sensors. Phys. Fluids, 10, 1182-1190.

[21] Protas, B., Bewley, T.R., \& Hagen, G. A comprehensive framework for the regularization of adjoint analysis in multiscale PDE systems. Submitted to Journal of Computational Physics. 
[22] Rathnasingham, R., \& Breuer, K.S. 1997 System identification and control of a turbulent boundary layer. Phys. Fluids, 9, 1867-1869.

[23] RÜtten, M., \& Dallmann, U. 2001 Numerische Simulation des Wirbelplatzens über einem Deltaflügel zur Identifikation von Beeinflussungsmaßnahmen. Z Angew. Math. Mech., 81, S423-S424, Suppl. 2.

[24] SAut, J.-C., \& SCHEURER, B. 1987 Unique continuation for some evolution equations. J. Differential Equations, 66, 118-139.

[25] Theofilis, V., Hein, S., \& Dallmann, U. 2000 On the origins of unsteadiness and threedimensionality in a laminar separation bubble. Phil. Trans. of the Royal Society London, Series A, 358, 3229-3246.

[26] Yoshino, T., Tsuda, M., Suzuki, Y., \& Kasagi, N. 2002 Toward development of feedback control system for wall turbulence with MEMS sensors and actuators. Proceedings of the 3rd Symposium on Smart Control of Turbulence, Tokyo, p. 115-120. 